\title{
Concomitant xanthogranulomatous pyelonephritis with renal abscess - an unusual cause of a right flank mass
}

\author{
Valencia Long ${ }^{1}$, Young Hwa Soon ${ }^{1}$, Michelle Rui Ting Soo ${ }^{1}$, Li Feng Tan² \\ ${ }^{1}$ Fast Program, Alexandra Hospital, National University Hospital System, Singapore; ${ }^{2}$ Division of \\ Healthy Ageing, National University Hospital System, Alexandra Hospital, Singapore
}

\section{INTRODUCTION}

Xanthogranulomatous

pyelonephritis (XGP) is an uncommon chronic granulomatous renal infection that can result in loss of renal function. Normal renal tissue is replaced by xanthogranulomatous material and serosanguinous fluid filled cysts infiltrated by lipid-laden macrophages (foam cells) (1). Women are more commonly affected than men (1).

We present a frail elderly lady with an unusual right sided flank mass, due to concomitant xanthogranulomatous pyelonephritis and renal abscess.

\section{CASE PRESENTATION}

A frail, 89-year-old female presented with progressive functional decline and loss of appetite, after she had an unwitnessed fall 2 weeks ago.

Past medical history was significant for well-controlled hypertension, osteoporosis, and cognitive impairment. She was febrile (temperature 38 degrees Celsius), blood pressure $110 \mathrm{mmHg} / 80 \mathrm{mmHg}$, pulse rate 85 beats per minute, saturating $100 \%$ on ambient air. Physical examination revealed a $5 \times 5 \mathrm{~cm}$ soft, right flank mass protruding from the posterolateral aspect of the right flank (Figure-1A).

Laboratory studies revealed leucocytosis $\left(15.8 \times 10^{9} / \mathrm{L}\right)$. She had pyuria on urine microscopy.
Renal function was normal. She was treated empirically with intravenous co-amoxiclav for urinary tract infection.

A Computed Tomography (CT) of the abdomen and pelvis performed to evaluate the right flank mass revealed bilateral renal calculi and dilated calyces at the lower poles of both kidneys representing focal xanthogranulomatous pyelonephritis (XPG). On the left, the inflammatory changes extended into the perirenal fat (stage II XPG). On the right, these changes extended more extensively into the retroperitoneum and herniated through the posterior abdominal wall (stage III XPG), with concomitant renal abscess. Rim-enhancement of these cystic structures with surrounding mild fat stranding was seen, representing acute superimposed infection (Figure-1B, Axial View of the CT scan: white asterisk representing right sided stage III XPG, white diamond representing the renal abscess herniating through the posterior abdominal wall, Figure-1C, Sagittal View of the CT scan: white asterisk representing right sided stage III XPG, white diamond representing renal abscess).

She was referred to Urology and underwent an ultrasound-guided percutaneous drainage with two interlocking drains inserted for the right renal abscess located at the right inferior renal pole respectively. Fluid culture revealed Streptococcus anginosus. Cytology was compatible with acute suppurative inflammation. Urine culture revealed 
Figure 1 - An 89 year-old female presenting with functional decline and a fall was discovered to have a right flank mass. She underwent CT abdomen and pelvis to evaluate the flank mass.

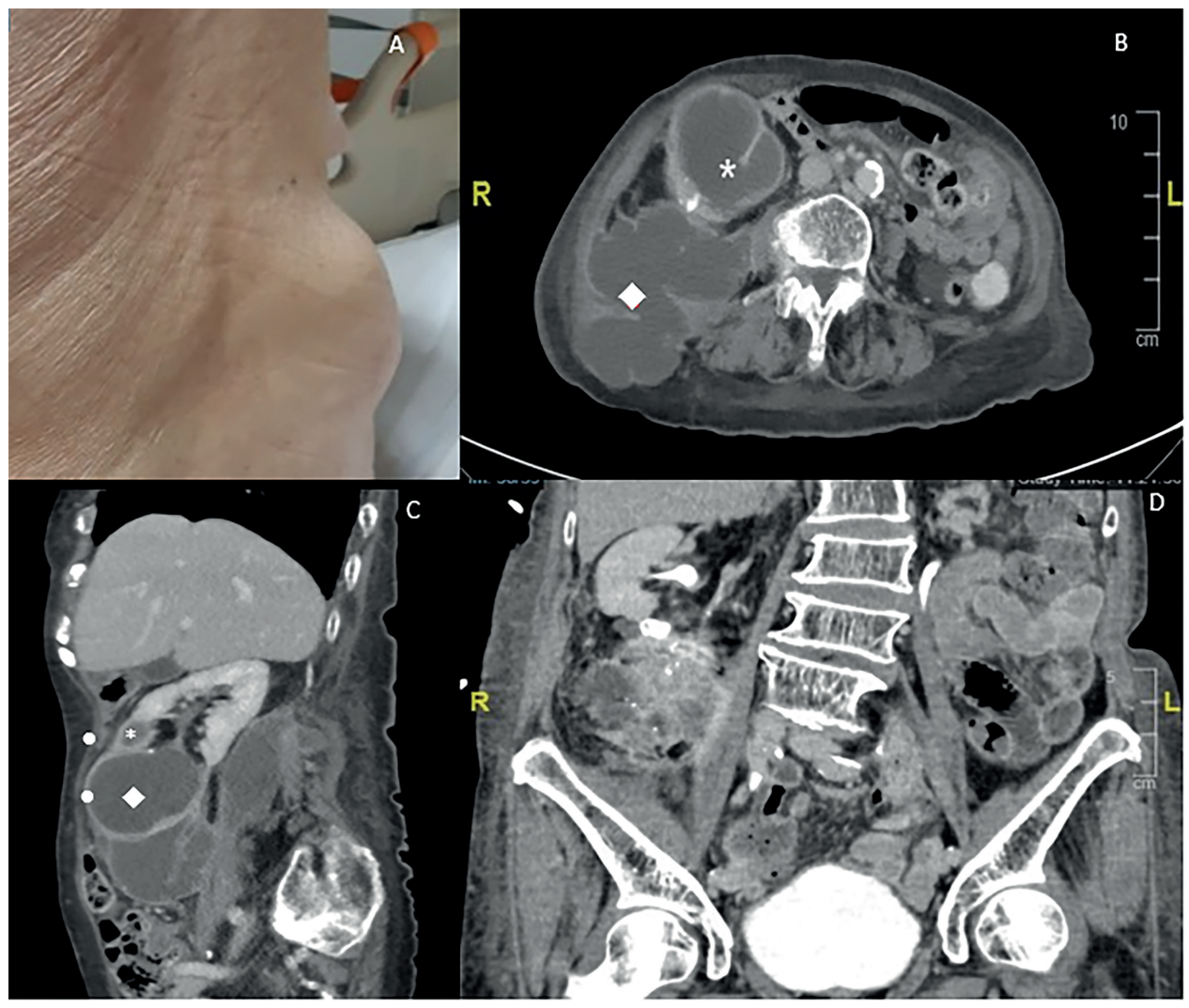

A) Side view of right flank mass; B) Axial section of CT abdomen and pelvis showing right sided stage III XPG (white asterisk), renal abscess (white diamond); 1C) Sagittal section of CT abdomen and pelvis (white asterisk representing the stage III XPG, white diamond representing renal abscess); D) Excretory phase of CT urogram showing lack of contrast flow through the renal abscess.

no growth. The drain was removed subsequently after a CT urogram confirmed near resolution of the collections. The excretory phase of the CT urogram confirmed that there was no passage of contrast through the renal abscess (Figure-1D). The patient remained stable and was eventually discharged with a one-month course of oral co-amoxiclav. There was no recurrence of the renal abscess.

\section{DISCUSSION}

We present an uncommon cause of a right sided flank mass in a patient initially admitted for a fall, and then evaluated to have bilateral xanthogranulomatous pyelonephritis (XGP) with concomitant right sided renal abscess formation.
XGP can present acutely with urinary tract infection symptoms including dysuria, haematuria, fever. In chronic cases, non-specific symptoms of weight loss, malaise can arise. Causes of XGP include chronic renal obstruction, infection, abnormal lipid metabolism, lymphatic obstruction and renal ischemia. Abscess formation (as was observed in our patient), fistula formation (reno-cutaneous, reno-colonic) and profound sepsis are known complications (2).

Although the presenting symptoms of XGP may be similar to renal and perinephric abscesses, the imaging findings are distinct. Typical CT findings of XGP include renal enlargement and parenchymal inflammation. Multiple areas of low attenuation may be observed within the kidney, and these represent dilated renal calyces with pus-filled cavities repla- 
cing normal renal parenchyma. The characteristic thinning of the cortex associated with dilated calyces is also referred to as the "bear paw" sign (3). CT classification of XGP falls into 3 stages: stage I (nephric) is a localized disease confined to the renal parenchyma; stage II (perinephric) lesions involve perinephric fat; and stage III (paranephric) lesions extend beyond Gerota's fascia into the retroperitoneum (3). antimicrobials to achieve source control. Surgical treatment options include en-bloc nephrectomy, in tulas closed. In this patient with bilateral XGP, partial nephrectomy can be considered (4). Laparoscopic nephrectomy is an option, depending on the extent of the lesions and the experience of the managing urologist (5). Recurrent XGP may warrant partial or full nephrectomy.

The treatment for renal abscess arising as a complication of XGP usually involves antimicrobial therapy with percutaneous drainage especially when the abscess size is large $(>5 \mathrm{~cm})(6,7)$. When there is urological obstruction (such as obstructing calculus), the obstruction should be relieved. In cases where the abscess cannot be successfully treated with antibiotics and percutaneous drainage, surgical drainage may be warranted (8).

This patient was treated conservatively as she was frail and unfit for surgery. Percutaneous drainage was performed to decompress the renal abscess.

We emphasise that early awareness of the possible diagnosis and prompt investigations are important to avoid aggravation of the condition requiring more aggressive measures later on, especially in patients with possible predisposing factors.

\section{CONFLICT OF INTEREST}

None declared.
The treatment for XGP involves the use of which all the involved tissue is removed and any fis-

\section{ACKNOWLEDGE}

Law Yu Xi Terence, Department of Urology, National University Hospital, National University Health System, Singapore

For your dedication in reviewing the article.

\section{REFERENCES}

1. Fan CM, Whitman GJ, Chew FS. Xanthogranulomatous pyelonephritis. AJR Am J Roentgenol. 1995; 165:1008.

2. Kundu R, Baliyan A, Dhingra $H$, Bhalla V, Punia RS. Clinicopathological Spectrum of Xanthogranulomatous Pyelonephritis. Indian J Nephrol. 2019; 29:111-5.

3. Tan WP, Papagiannopoulos D, Elterman L. Bear's Paw Sign: A Classic Presentation of Xanthogranulomatous Pyelonephritis. Urology. 2015; 86:e5-6.

4. PerézLM, Thrasher JB, AndersonEE. Successfulmanagement of bilateral xanthogranulomatous pyelonephritis by bilateral partial nephrectomy. J Urol. 1993; 149:100-2.

5. Guzzo TJ, Bivalacqua TJ, Pierorazio PM, Varkarakis J, Schaeffer EM, Allaf ME. Xanthogranulomatous pyelonephritis: presentation and management in the era of laparoscopy. BJU Int. 2009; 104:1265-8.

6. Lee SH, Jung HJ, Mah SY, Chung BH. Renal abscesses measuring $5 \mathrm{~cm}$ or less: outcome of medical treatment without therapeutic drainage. Yonsei Med J. 2010; 51:569-73.

7. Coelho RF, Schneider-Monteiro ED, Mesquita JL, Mazzucchi E, Marmo Lucon A, Srougi M. Renal and perinephric abscesses: analysis of 65 consecutive cases. World J Surg. 2007; 31:431-6.

8. Fowler JE Jr, Perkins T. Presentation, diagnosis and treatment of renal abscesses: 1972-1988. J Urol. 1994; 151:847-51.

ARTICLE INFO

Valencia Long http://orcid.org/0000-0002-5445-5698

Int Braz J Urol. 2021; 47: 1069-71

Submitted for publication:

Valencia Long, MD

Fast Program,

Alexandra Hospital, National University

Hospital System, Singapore

5 Lower Kent Ridge Rd,

119074, Singapore

E-mail: valencialong@gmail.com

$$
\begin{array}{r}
\text { Submitted for publication: } \\
\text { October 07, 2020 } \\
\hline \text { Accepted after revision: } \\
\text { January 07, 2021 } \\
\text { Published as Ahead of Print: } \\
\text { April 20, 2021 }
\end{array}
$$

